\title{
Physician age and outcomes in elderly patients in hospital in the US: observational study
}

\author{
Yusuke Tsugawa, ${ }^{1,2}$ Joseph P Newhouse, ${ }^{1,3,4,5}$ Alan M Zaslavsky, ${ }^{3}$ Daniel M Blumenthal, ${ }^{6}$ \\ Anupam B Jena $3,5,7$
}

${ }^{1}$ Department of Health Policy and Management, Harvard T. H. Chan School of Public Health, Boston, MA, USA

${ }^{2}$ Division of General Internal Medicine and Primary Care,

Brigham and Women's Hospital,

Boston, MA, USA

${ }^{3}$ Department of Health Care

Policy, Harvard Medical School,

Boston, MA, USA

${ }^{4}$ Harvard Kennedy School,

Cambridge, MA, USA

${ }^{5}$ National Bureau of Economic

Research, Cambridge, MA, USA

${ }^{6}$ Cardiology Division,

Massachusetts General

Hospital, Boston, MA, USA

${ }^{7}$ Department of Medicine,

Massachusetts General

Hospital, Boston, MA, USA

Correspondence to: Y Tsugawa,

Harvard T. H. Chan School of

Public Health, Department of

Health Policy and Management,

42 Church Street, Cambridge,

MA 02138, USA

ytsugawa@hsph.harvard.edu

Additional material is published online only. To view please visit the journal online.

Cite this as: BMJ 2017;357:j1797 http://dx.doi.org/10.1136/bmj.j1797

Accepted: 6 March 2017

\section{ABSTRACT}

OBJECTIVES

To investigate whether outcomes of patients who were admitted to hospital differ between those treated by younger and older physicians.

DESIGN

Observational study.

SETTING

US acute care hospitals.

\section{PARTICIPANTS}

$20 \%$ random sample of Medicare fee-for-service beneficiaries aged $\geq 65$ admitted to hospital with a medical condition in 2011-14 and treated by hospitalist physicians to whom they were assigned based on scheduled work shifts. To assess the generalizability of findings, analyses also included patients treated by general internists including both hospitalists and non-hospitalists.

\section{MAIN OUTCOME MEASURES}

30 day mortality and readmissions and costs of care.

RESULTS

736537 admissions managed by 18854 hospitalist physicians (median age 41) were included. Patients' characteristics were similar across physician ages. After adjustment for characteristics of patients and physicians and hospital fixed effects (effectively comparing physicians within the same hospital),

\section{WHAT IS ALREADY KNOWN ON THIS TOPIC}

Whether quality of care differs between younger and older physicians remains largely unknown

Though clinical skills and knowledge accumulated by more experienced physicians could lead to improved quality of care, physicians' skills might become outdated as scientific knowledge, technology, and clinical guidelines change

Older physicians might have decreased clinical knowledge, adhere less often to standards of appropriate treatment, and perform worse on process measures of quality with respect to diagnosis, screening, and preventive care

\section{WHAT THIS STUDY ADDS}

This study examined patient outcomes, including 30 day mortality, readmissions, and costs of care, in a nationally representative sample of US Medicare beneficiaries admitted to hospital with a medical condition in 2011-14

Patients were treated by hospitalists (physicians whose clinical focus is caring of patients in hospital), to whom they are typically assigned based on scheduled work shifts

Within the same hospital, patients treated by older hospitalists had similar characteristics to patients treated by younger hospitalists but had higher mortality rates, with the exception of those hospitalists who treated high volumes of patients Readmissions did not vary with physician age, while costs of care were slightly higher among older physicians patients' adjusted 30 day mortality rates were $10.8 \%$ for physicians aged $<40$ (95\% confidence interval $10.7 \%$ to $10.9 \%), 11.1 \%$ for physicians aged $40-49$ (11.0\% to $11.3 \%), 11.3 \%$ for physicians aged $50-59$ (11.1\% to $11.5 \%$ ), and $12.1 \%$ for physicians aged $\geq 60$ (11.6\% to $12.5 \%)$. Among physicians with a high volume of patients, however, there was no association between physician age and patient mortality. Readmissions did not vary with physician age, while costs of care were slightly higher among older physicians. Similar patterns were observed among general internists and in several sensitivity analyses.

CONCLUSIONS

Within the same hospital, patients treated by older physicians had higher mortality than patients cared for by younger physicians, except those physicians treating high volumes of patients.

\section{Introduction}

The relation between physician age and performance remains largely unknown, particularly with respect to patient outcomes. Clinical skills and knowledge accumulated by more experienced physicians can lead to improved quality of care. Physicians' skills, however, can also become outdated as scientific knowledge, technology, and clinical guidelines change. Incorporating these changes into clinical practice is time consuming and can at times be overwhelming. ${ }^{1-3}$ Interest in how quality of care evolves over a physician's career has revived in recent years, with debates over how best to structure programs for continuing medical education, including recent controversy in the US regarding maintenance of certification programs.

A systematic review of the relation between physician experience and quality of care found that older physicians might perform worse-older physicians have decreased clinical knowledge, adhere less often to standards of appropriate treatment, and perform worse on process measures of quality with respect to diagnosis, screening, and preventive care. ${ }^{4}$ Data on patient outcomes, which arguably are most important, have been scarce. ${ }^{4}$ Existing studies have also been limited in size or disease scope and have not been nationally representative..$^{5-7}$ As a result, whether physician age is associated with patient outcomes remains largely unknown.

Using nationally representative data on Medicare beneficiaries admitted to hospital with a medical condition during 2011-14, we sought answers to three questions. First, what is the association between age of the treating physician and patient mortality after admission? Second, does this association vary with the volume of patients a physician treats? Finally, given 
national efforts to improve the efficiency of healthcare, is physician age associated with readmissions and costs of care?

\section{Methods \\ Data}

We linked multiple data sources: the 20\% Medicare Inpatient Carrier and Medicare Beneficiary Summary Files (2011-14); physician data collected by Doximity (an online professional network for physicians); and the American Hospital Association (AHA) annual survey of hospital characteristics (2012). Doximity has assembled data on all US physicians (both those who are registered members of the service as well as those who are not) from multiple sources and data partnerships, including the national plan and provider enumeration system national provider identifier registry, state medical boards, specialty societies such as the American Board of Medical Specialties, and collaborating hospitals and medical schools. The database includes information on physician age, sex, year of completion and name of medical school, residency, and board certification..$^{8-12}$ Previous studies have validated data for a random sample of physicians in the Doximity database by using manual audits. ${ }^{89}$ We were able to match about $95 \%$ of physicians in the Medicare database to the Doximity database.

\section{Patients}

We identified beneficiaries of Medicare fee-for-service aged $\geq 65$ who were admitted to hospital with a medical condition (as defined by the presence of a medical diagnosis related group on admission) from 1 January 2011 to 31 December 2014. We restricted our sample to patients treated in acute care hospitals and excluded elective admissions and those in which a patient left against medical advice. To allow sufficient follow-up, we excluded patients admitted in December 2014 from 30 day mortality analyses and patients discharged in December 2014 from readmission analyses.

\section{Medicare hospital spending and method of} assigning physicians to patients

In the US, Medicare spending on patients in hospital mainly consists of two components: parts A and B. Part A spending is a fixed payment to a hospital per patient that is determined by the final diagnosis or diagnoses of the patient (categorized into diagnosis related groups) and broadly reflects hospital costs other than professional services. Within each hospital the part A payment does not vary for patients within the same diagnosis related group (with a few exceptions). Part B uses fee-for-service payment, and spending varies with the intensity of services delivered, including visits, procedures, and interpretation of tests and images. Based on previous studies, ${ }^{10-12}$ we defined the responsible physician for a given admission as the physician who billed the largest share of part B costs during that admission..$^{13}$ In a sensitivity analysis, we used alternative assignment methods to assess the robustness of our findings to this attribution rule.
We restricted our analyses to admissions for which the highest spending physicians were hospitalists (described below) or general internists. For patients transferred to other acute care hospitals (1.2\% of admissions), we attributed the multi-hospital episode of care and associated outcomes to the assigned physician of the initial admissions. ${ }^{1415}$ On average, 51\%, 22\%, and $11 \%$ of total part B spending was accounted for by the first, second, and third highest spending physicians, respectively.

Our primary analysis focused on patients treated by hospitalists to examine the possibility that older physicians might treat patients with greater or lesser unmeasured severity of illness. Hospitalists are physicians whose clinical focus is caring for patients admitted to hospital. ${ }^{1617}$ They are typically trained in internal or family medicine. Some complete subspecialty training as well (such as infectious disease or nephrology) but decide to practice general inpatient medicine. The hospitalist specialty began in the 1990s in the US and is the most rapidly growing medical specialty there. Before the introduction of hospitalists, a patient admitted for a general medical condition was cared for by that patient's primary care physician (equivalent to general practitioner in the UK), who, on any given day, would typically visit his/her inpatients when time permitted in the outpatient schedule. In 2016, it was estimated that more than 50000 hospitalists were practicing in the US, and about $75 \%$ of US hospitals now have hospitalists. ${ }^{18}$

Hospitalists typically work in scheduled shifts or blocks (such as one week on and one week off) and do not treat patients in the outpatient setting. Therefore, within the same hospital, patients treated by hospitalists are plausibly quasi-randomized to a particular hospitalist based only on the time of the patient's admission and the hospitalist's work schedule. ${ }^{1011}$ We assessed the validity of this assumption by testing the balance of a broad range of patient characteristics across categories of age of hospitalist. We defined hospitalists as general internists who filed at least $90 \%$ of their total evaluation and management billings in an inpatient setting, a claims based approach that a previous study validated by calling physicians to confirm that they were indeed hospitalists (sensitivity of 84.2\%, specificity of $96.5 \%$, and a positive predictive value of $88.9 \%)^{20}$

\section{Physician age}

Physician age was defined as the age on the date of admission of patients. Data on physician age were available for $93.5 \%$ of physicians. Physician age was modeled both as a continuous linear variable and as a categorical variable (in categories of $<40,40-49,50-59$, and $\geq 60$ ) to allow for a potential non-linear relation with patient outcomes. We also used linear spline models.

\section{Patient outcomes}

The primary outcome was the 30 day mortality rate in patients (death within 30 days of admission); secondary outcomes were 30 day readmission rates (readmission within 30 days of discharge) and costs of care. 
Information on dates of death, including deaths out of hospital, was available in the Medicare Beneficiary summary files. Over $99 \%$ of dates of death in these files have been verified by death certificate. ${ }^{21}$ For mortality analyses, we excluded patients whose death dates were not validated. We defined costs of care as total part B spending per admission.

\section{Adjustment variables}

We adjusted for patient characteristics, physician characteristics, and hospital fixed effects. Patient characteristics included age in five year increments, sex, race or ethnic group (non-Hispanic white, non-Hispanic black, Hispanic, other), primary diagnosis (diagnosis related group), 27 comorbidities (Elixhauser comorbidity index ${ }^{22}$ ), median household income of zip code (in 10ths), an indicator for dual Medicare-Medicaid coverage, day of the week of the admission date (to account for the possibility that severity of illness of patients could be higher on specific days of the week), and year indicators. Physician characteristics (other than age) included sex, indicator variables for medical school from which a physician graduated (all foreign schools were grouped into a single category), and whether they graduated from allopathic (MD) or osteopathic (DO) medical schools (allopathic and osteopathic schools both teach the same basic curriculums necessary to become a qualified physician, but osteopathic schools emphasize prevention and other techniques as well). We included indicator variables for each hospital, which allowed each hospital to have its own intercept in the regression analyses, a statistical method known as hospital fixed effects. Hospital fixed effects account for both measured and unmeasured characteristics of hospitals that do not vary over time, including unmeasured differences in patient populations, thereby effectively comparing patient outcomes among hospitalists of varying age within the same hospital. ${ }^{23-25}$

\section{Statistical analysis}

First, we examined the association between physician age and 30 day mortality using a multivariable logistic regression model treating age as both a continuous variable and a categorical variable to allow for a non-linear relation, adjusting for patient and physician characteristics and hospital fixed effects. We also used linear age splines. To evaluate whether splines improve goodness of fit compared with modeling a linear relation between physician age and patient mortality, we performed a Wald test adjusted for clustering (to approximate a likelihood ratio test because standard likelihood based tests are unavailable with clustered data). To account for potential correlations of patient outcomes within the same physicians, we clustered standard errors at the physician level. ${ }^{26}$ To overcome complete or quasi-complete separation problems (perfect or nearly perfect prediction of the outcome by the model), we combined diagnosis related group codes with no outcome event (30 day mortality or readmission) into clinically similar categories. ${ }^{27}$ We calculated adjusted 30 day mortality rates using margins of responses (also known as predictive margins); for each admission we calculated predicted probabilities of outcome with physician age group fixed at each level and then averaged over the distribution of covariates in our national sample. ${ }^{28}$

Second, because physicians with high volumes of patients might better maintain clinical knowledge and skills, ${ }^{29-32}$ we examined whether the association between physician age and patient mortality was modified by volume. We classified physicians into thirds of patient volume: low (estimated number of total admissions $<90$ per year), medium (91-200 admissions), and high (>201 admissions). Within each group, we examined the association between physician age and patient mortality, adjusting for patient and physician characteristics and hospital fixed effects. We used a Wald test to formally test the interaction between physician age and patient volume.

Finally, we evaluated the association between physician age and 30 day readmissions and costs of care. We used multivariable logistic regression models for readmission analyses. Because cost data were right skewed, we used a generalized linear model (GLM) with a log link and gamma distribution. ${ }^{33}$

\section{Secondary analyses}

We conducted several secondary analyses. First, to test the generalizability of our findings, we repeated our analyses among general internists overall, including both hospitalists and non-hospitalists. Second, to evaluate whether our findings were sensitive to how we attributed patients to physicians, we tested two alternative attribution rules: attributing patients to physicians with the largest number of evaluation and management claims and attributing patients to physicians who billed the first claim for a given admission ("admitting physician"). Third, because the association between physician age and mortality could be confounded by unobserved care preferences of patients, such as do-not-resuscitate directives, we excluded patients with cancer and those discharged to a hospice. Fourth, to assess the relation between physician age and patient outcomes in a relatively young population whose probability of death is lower, we restricted our analysis to patients aged 65-75. Fifth, an increasing number of young subspecialists in specialties like nephrology and infectious disease work as hospitalists but were excluded from our primary analyses. To investigate this, we reanalyzed the data including hospitalists with medical subspecialties and adjusted for their specialty. Sixth, patients who are admitted multiple times might not be randomly assigned to a given hospitalist but instead to the hospitalist who treated the patient previously. To deal with this, we reanalyzed the data after restricting our sample to the first admission. Seventh, we also evaluated in hospital, 60 day, and 90 day mortality rates to assess if any survival gains were short lived. Eighth, we used generalized estimating equations (GEE) with an independent covariance matrix to account for the hierarchical structure of the data because of the grouping of patients within hospitals, adjusting for 
patient and physician characteristics and hospital fixed effects. ${ }^{34}$ Ninth, to focus on more homogenous patient populations, we separately analyzed the four most common conditions treated by hospitalists in our data (sepsis, pneumonia, congestive heart failure, and chronic obstructive pulmonary disease) (see table $\mathrm{A}$ in the appendix for diagnosis codes). Tenth, we used years since completion of residency, instead of physician age, as a measure of physician experience. We did not use this variable for our primary analyses because data on year of residency completion were missing for 35.5\% of physicians, and we were concerned that missingness might not be at random. Eleventh, we conducted a formal sensitivity analysis to assess the extent to which an unmeasured confounder might explain our results. ${ }^{35}$ Twelfth, we conducted cost analysis using different model specifications: a GLM model with a log link and a negative binomial distribution, a GLM model with a log link and a Poisson distribution, and an ordinary least squares model after winsorizing the top $1 \%$ of observations with largest residuals (replacing outlier costs by the most extreme retained values). Finally, we conducted analyses among subgroups including Medicare beneficiaries aged $\geq 65$ who were admitted to hospital with an emergency medical condition (as opposed to our baseline analysis of "non-elective" conditions, which included both emergency and urgent admissions), Medicare beneficiaries aged $\geq 65$ who were admitted with an elective medical condition, and Medicare beneficiaries aged 20-64. The latter group qualified for Medicare through disability and has generally worse health status than the general US population aged below 65, but nonetheless the generalizability of our findings to populations of younger patients is of interest.

Data preparation was conducted with SAS, version 9.4 (SAS Institute), and analyses were performed with Stata, version 14 (Stata-Corp, College Station, TX).

\section{Patient involvement}

No patients were involved in setting the research question or the outcome measures, nor were they involved in developing plans for the design or implementation of the study. No patients were asked to advise on interpretation or writing up of results. There are no plans to disseminate the results of the research to study participants or the relevant patient community. Patient consent was not required for the study.

\section{Results}

\section{Physician and patient characteristics}

The median and mean age among 18854 hospitalist physicians in our sample in 2014 was 41.0 and 42.9, respectively. A broad range of patient characteristics, including the number of Elixhauser comorbidities and composite Elixhauser comorbidity scores, ${ }^{36}$ were balanced across physicians with different ages (table 1).

\begin{tabular}{|c|c|c|c|c|c|}
\hline & \multicolumn{5}{|c|}{ Physician age (years) } \\
\hline & $<40(n=10177)$ & $40-49(n=8016)$ & $50-59(n=3331)$ & $\geq 60(n=1086)$ & P value \\
\hline \multicolumn{6}{|l|}{ Physician characteristics } \\
\hline Mean (SD) age (years) & $35.3(2.6)$ & $43.89(2.8)$ & $53.8(2.9)$ & $63.7(3.5)$ & $<0.001$ \\
\hline Women & 39.0 & 34.4 & 28.7 & 15.7 & $<0.001$ \\
\hline Mean (SD) years of experience since completion of residency & $4.9(2.7)$ & $10.9(4.3)$ & $18.1(6.7)$ & $28.6(8.6)$ & $<0.001$ \\
\hline MD (allopathic) & 93.4 & 95.1 & 95.2 & 97.5 & $<0.001$ \\
\hline Annual No of admissions/physician* & 136 & 156 & 155 & 127 & $<0.001$ \\
\hline \multicolumn{6}{|l|}{ Patient characteristics } \\
\hline No of Medicare admissions in 2011-13 & 309020 & 280894 & 115660 & 30963 & \\
\hline Mean (SD) age (years) & $80.5(8.5)$ & $80.6(8.5)$ & $80.5(8.5)$ & $80.6(8.6)$ & 0.01 \\
\hline Women & 60.7 & 60.5 & 60.3 & 60.3 & 0.40 \\
\hline \multicolumn{6}{|l|}{ Race: } \\
\hline White & 82.6 & 82.6 & 83.2 & 83.2 & 0.72 \\
\hline Black & 10.2 & 9.7 & 9.4 & 9.4 & 0.53 \\
\hline Hispanic & 4.3 & 4.7 & 4.7 & 4.5 & 0.47 \\
\hline Other & 3.0 & 3.0 & 2.7 & 3.0 & 0.31 \\
\hline Median household income (\$) & 58240 & 57858 & 55133 & 53167 & 0.06 \\
\hline Medicaid status & 22.6 & 23.3 & 24.2 & 25.5 & 0.01 \\
\hline No of Elixhauser comorbidities & 3.4 & 3.4 & 3.4 & 3.4 & $<0.001$ \\
\hline Elixhauser comorbidity score ${ }^{\dagger}$ & 6.7 & 6.7 & 6.8 & 6.9 & $<0.001$ \\
\hline \multicolumn{6}{|l|}{ Comorbidities: } \\
\hline Congestive heart failure & 19.8 & 19.7 & 19.7 & 20.3 & $<0.001$ \\
\hline Chronic obstructive pulmonary disease & 24.4 & 25.2 & 26.1 & 27.1 & $<0.001$ \\
\hline Diabetes & 31.5 & 31.5 & 32.0 & 32.3 & 0.44 \\
\hline Renal failure & 22.5 & 22.0 & 22.2 & 22.3 & 0.88 \\
\hline Neurological disorders & 15.3 & 15.5 & 16.2 & 16.8 & $<0.001$ \\
\hline Cancer & 7.2 & 7.0 & 6.9 & 7.3 & 0.03 \\
\hline Mental illness & 15.2 & 15.0 & 15.4 & 15.8 & 0.14 \\
\hline
\end{tabular}

*Estimated using facts that our sample was $20 \%$ random sample of fee-for-service Medicare beneficiaries, that proportion of all Medicare beneficiaries with Medicare Advantage plans is about $30 \%$, and that Medicare beneficiaries (including fee-for-service plus Medicare Advantage) comprise $40 \%$ of all hospital admissions in US. See appendix for details.

tComposite score developed by van Walraven et a ${ }^{36}$ was used to combine Elixhauser comorbidities into single numeric score. 


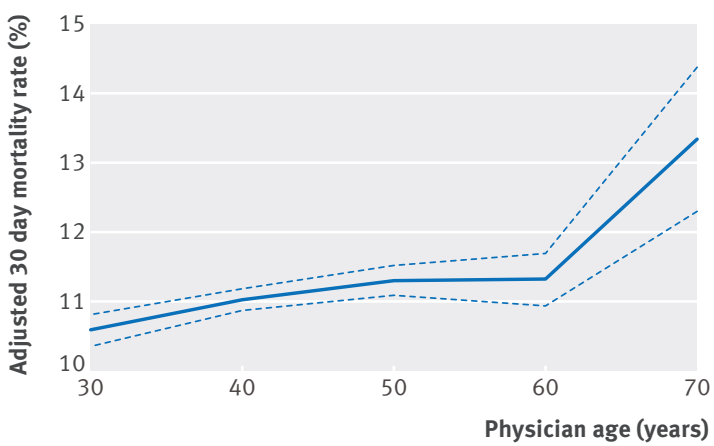

Fig 1 | Adjusted association between physician age and patient mortality with linear spline model. Multivariable logistic regression model with linear splines was used with knots placed at physician age of 40,50 , and 60 , adjusted for patient and physician characteristics and hospital fixed effects. Solid line represents point estimates, and shaded area represents $95 \% \mathrm{Cl}$ around these estimates

Physician age and patient mortality

The overall 30 day mortality rate in our final sample of 736537 hospital admissions was $11.1 \%$. Figure 1 shows the results of a logistic regression model with linear splines. After adjustment for patient and physician characteristics and hospital fixed effects, older physicians had significantly higher patient mortality than younger physicians. We could reject the null of linearity ( $\mathrm{P}=0.02$, fig 1). Because we observed a non-linear relation between physician age and patient mortality, we also fitted a linear spline logistic model allowing for different slopes for physicians aged $<60$ and aged $\geq 60$ and found that an additional 10 years increase in physician age was associated with an adjusted odds ratio of 30 day mortality of 1.03 (95\% confidence interval 1.02 to 1.05; $\mathrm{P}<0.001$ ) for physicians aged $<60$, and 1.22 (1.08 to 1.37; $\mathrm{P}=0.01$ ) for physicians aged $\geq 60$ (table 2 ). When age was modeled as a continuous linear variable, an additional 10 year increase in physician age was associated with an adjusted odds ratio of 30 day mortality of

Table 2 | Association between physician age and 30 day patient mortality in patients admitted to hospital. Table includes results of three analyses: modeling age as a continuous variable, modeling age as a continuous variable with separate splines at ages $<60$ and $\geq 60$, and modeling age as categorical variable. All models adjusted for patient and physician characteristics and hospital fixed effects. Standard errors were clustered at physician level. Estimates should be interpreted as average odds ratio across all physician age categories

\begin{tabular}{|c|c|c|c|c|}
\hline Physician age & $\begin{array}{l}\text { No of admissions } \\
\text { (No of physicians*) }\end{array}$ & $\begin{array}{l}\text { Adjusted } 30 \text { day } \\
\text { mortality rate }(95 \% \mathrm{Cl})\end{array}$ & $\begin{array}{l}\text { Adjusted odds ratio } \\
(95 \% \mathrm{Cl})\end{array}$ & P value \\
\hline \multicolumn{5}{|c|}{ Physician age (years) as continuous variable (for every 10 years) } \\
\hline Overall & $736537(18854)$ & - & $1.04(1.03$ to 1.06$)$ & $<0.001$ \\
\hline$<60$ & $705574(18180)$ & - & $1.03(1.02$ to 1.05$)$ & $<0.001$ \\
\hline 60 & $30963(1086)$ & - & $1.22(1.08$ to 1.37$)$ & 0.001 \\
\hline
\end{tabular}

\begin{tabular}{|lllll}
\hline \multicolumn{4}{l}{ Physician age (years) as categorical variable } & \\
\hline$<40$ & $309020(10177)$ & $10.8 \%(10.7 \%$ to $10.9 \%)$ & Reference & - \\
\hline $40-49$ & $280894(8016)$ & $11.1 \%(11.0 \%$ to $11.3 \%)$ & $1.04(1.02$ to 1.07$)$ & $<0.001$ \\
\hline $50-59$ & $115660(3331)$ & $11.3 \%(11.1 \%$ to $11.5 \%)$ & $1.07(1.03$ to 1.10$)$ & $<0.001$ \\
\hline$\geq 60$ & $30963(1086)$ & $12.1 \%(11.6 \%$ to $12.5 \%)$ & $1.17(1.11$ to 1.23$)$ & $<0.001$ \\
\hline
\end{tabular}

${ }^{\star}$ Total number of physicians varies between continuous and categorical analyses because some physicians moved to higher age category during study period.

tEstimates should be interpreted as an average odds ratio across all physician age categories.
1.04 (1.03 to 1.06; $\mathrm{P}<0.001$ ), interpreted as the average odds ratio across all physician age groups (table 2 ).

Treating physician age as a categorical variable showed a monotonic relation between physician age and patient mortality. Physicians aged $<40$ had the lowest patient mortality rate (adjusted 30 day mortality rate $10.8 \%$, 95\% confidence interval $10.7 \%$ to $10.9 \%$ ), followed by physicians aged $40-49$ (11.1\%, $11.0 \%$ to $11.3 \%)$, $50-59(11.3 \%, 11.1 \%$ to $11.5 \%)$, and $\geq 60(12.1 \%, 11.6 \%$ to $12.5 \%$ ) (table 2).

\section{Physician age and patient mortality by volume}

Physician age was positively associated with patient mortality among physicians with low and medium volumes of patients but not among those with high volumes of patients (table 3), who also had the lowest overall mortality rate. For instance, each 10 year increase in physician age was associated with adjusted odds ratios of 30 day mortality of 1.19 (95\% confidence interval 1.14 to 1.23; $\mathrm{P}<0.001$ ) and 1.06 (1.03 to 1.09; $\mathrm{P}<0.001)$ among low and medium volume physicians, respectively. In contrast, despite the larger sample size among high volume physicians, we observed no association between physician age and patient mortality (adjusted odds ratio for additional 10 years, 1.01, 0.99 to 1.03; $\mathrm{P}=0.29)$. The interaction between physician age and patient volume was significant $(\mathrm{P}<0.001)$.

\section{Patient readmissions and part $B$ spending}

We found no association between physician age and the patient 30 day readmission rate (adjusted odds ratio for additional 10 years, 1.00, 95\% confidence interval 0.99 to 1.01; $\mathrm{P}=0.82$ ) (table 4). Although differences in part $\mathrm{B}$ spending between physicians of varying age were significant, they were small. Each 10 year increase in physician age was associated with a $2.4 \%$ increase $(2.0 \%$ to $2.8 \% ; \mathrm{P}<0.001)$ in part B spending.

\section{Secondary analyses}

Our overall findings were qualitatively unaffected by including non-hospitalist general internists, although the observed relation between patient mortality and physician age was smaller (table B in appendix). The smaller relation between physician age and patient outcomes might be because of unobserved differences in severity of illness between patients treated by young versus old physicians or actual differences in how physician age relates to patient mortality among hospitalists versus non-hospitalist general internists. Our findings were also not sensitive to using alternative methods for attributing physicians (table $\mathrm{C}$ in appendix); excluding patients with cancer or discharged to hospice (table D in appendix); restricting analysis to patients aged 65-75 (table $\mathrm{E}$ in appendix); including hospitalists with medical subspecialty boards (table $\mathrm{F}$ in appendix); restricting to the first admission for patients with multiple admissions (table $\mathrm{G}$ in appendix); using in hospital, 60 day, and 90 day mortality rates instead of 30 day mortality (tables $\mathrm{H}$ and $\mathrm{I}$ in appendix); and the use of GEE instead of cluster robust standard errors (table J in appendix). When we stratified by primary 


\begin{tabular}{|c|c|c|c|c|}
\hline Physician age & $\begin{array}{l}\text { No of admissions } \\
\text { (No of physicians") }\end{array}$ & $\begin{array}{l}\text { Adjusted } 30 \text { day mortality } \\
\text { rate }^{\dagger}(95 \% \mathrm{Cl})\end{array}$ & $\begin{array}{l}\text { Adjusted odds ratio }{ }^{\dagger} \\
(95 \% \mathrm{Cl})\end{array}$ & $P$ value \\
\hline \multicolumn{5}{|c|}{ Low volume physicians ( $<90$ cases/year $)$} \\
\hline Continuous ${ }^{\S}$ & $62634(6246)$ & - & 1.19 (1.14 to 1.23$)$ & $<0.001$ \\
\hline$<40$ & $35540(3666)$ & $12.7 \%(12.3 \%$ to $13.0 \%)$ & Reference & - \\
\hline $40-49$ & $16988(2070)$ & $14.6 \%(14.0 \%$ to $15.1 \%)$ & $1.24(1.15$ to 1.34$)$ & $<0.001$ \\
\hline $50-59$ & 7259 (941) & $16.0 \%(15.1 \%$ to $16.8 \%)$ & $1.44(1.30$ to 1.60$)$ & $<0.001$ \\
\hline$\geq 60$ & $2847(388)$ & $17.0 \%(15.5 \%$ to $18.4 \%)$ & 1.59 (1.36 to 1.85$)$ & $<0.001$ \\
\hline \multicolumn{5}{|c|}{ Medium volume physicians $\left(90-200{\left.\text { cases} / \text { year }^{\ddagger}\right)}^{\prime}\right.$} \\
\hline Continuous§ & $189902(6208)$ & - & $1.06(1.03$ to 1.09$)$ & $<0.001$ \\
\hline$<40$ & $95824(3521)$ & $10.8 \%(10.6 \%$ to $11.0 \%)$ & Reference & - \\
\hline $40-49$ & $63471(2622)$ & $11.2 \%(11.0 \%$ to $11.5 \%)$ & $1.06(1.01$ to 1.11$)$ & 0.01 \\
\hline $50-59$ & $23031(986)$ & $11.2 \%(10.8 \%$ to $11.7 \%)$ & 1.06 (0.99 to 1.13$)$ & 0.10 \\
\hline$\geq 60$ & $7574(318)$ & $12.5 \%(11.6 \%$ to $13.4 \%)$ & $1.23(1.10$ to 1.38$)$ & $<0.001$ \\
\hline \multicolumn{5}{|c|}{ High volume physicians ( $>201$ cases/year ${ }^{\ddagger}$ ) } \\
\hline Continuous $\$$ & $477627(6235)$ & - & $1.01(0.99$ to 1.03$)$ & 0.29 \\
\hline$<40$ & $174760(2907)$ & $10.7 \%(10.6 \%$ to $10.8 \%)$ & Reference & - \\
\hline $40-49$ & $198363(3251)$ & $10.9 \%(10.8 \%$ to $11.1 \%)$ & $1.03(1.004$ to 1.06$)$ & 0.03 \\
\hline 50-59 & $84402(1362)$ & $10.8 \%(10.6 \%$ to $11.1 \%)$ & $1.02(0.98$ to 1.05$)$ & 0.33 \\
\hline$\geq 60$ & $20102(365)$ & $10.9 \%(10.4 \%$ to $11.4 \%)$ & $1.02(0.96$ to 1.09$)$ & 0.51 \\
\hline
\end{tabular}

*Total number of physicians varies between continuous and categorical analyses because some physicians moved to higher age category during study period.

tAdjusted for patient and physician characteristics and hospital fixed effects. Standard errors were clustered at physician level.

¥Total number of admissions per physician estimated: our data were $20 \%$ random sample of fee-for-service Medicare beneficiaries, Medicare patients overall account for about $40 \%$ of all admissions because of medical conditions in the US, and about $70 \%$ of all Medicare beneficiaries (including fee-for-service Medicare and Medicare Advantage) are covered by fee-for-service Medicare.

§Estimates from continuous models should be interpreted as average odds ratio across all physician age categories.

diagnosis, older hospitalists had higher patient mortality for sepsis, congestive heart failure, and chronic obstructive pulmonary disease, but not for pneumonia (table $\mathrm{K}$ in appendix). When using years in practice instead of age we found similar results (table $\mathrm{L}$ in appendix). A formal test for an unmeasured confounder showed that it is unlikely that this could explain the observed association between physician age and patient mortality (table $\mathrm{M}$ in appendix). Use of different model specifications for cost analyses did not qualitatively affect our findings (table $\mathrm{N}$ in appendix). Finally, we observed similar relations between physician age and 30 day patient mortality in subgroup analyses of Medicare beneficiaries aged $\geq 65$ who were admitted with an emergency medical condition, Medicare beneficiaries aged $\geq 65$ who were admitted with an elective medical condition, and admitted Medicare beneficiaries aged 20-64 (tables $\mathrm{O}$ and $\mathrm{P}$ in appendix).

\section{Discussion}

\section{Principal findings}

In a national sample of elderly Medicare beneficiaries admitted to hospital with medical conditions, we found that patients treated by older physicians had higher

\begin{tabular}{|c|c|c|c|c|}
\hline Physician age & $\begin{array}{l}\text { No of admissions } \\
\text { (No of physicians") }\end{array}$ & $\begin{array}{l}\text { Adjusted patient } \\
\text { outcomes }^{\dagger}(95 \% \mathrm{Cl})\end{array}$ & $\begin{array}{l}\text { Adjusted odds ratio or } \\
\text { difference }{ }^{\dagger}(95 \% \mathrm{Cl})\end{array}$ & $\begin{array}{l}P \\
\text { value }\end{array}$ \\
\hline \multicolumn{5}{|c|}{30 day readmission rate } \\
\hline \multicolumn{5}{|l|}{ Continuous": } \\
\hline For every 10 years & 722999 (18854) & - & 1.00 (0.99 to 1.01$)$ & 0.82 \\
\hline \multicolumn{5}{|l|}{ Categorical: } \\
\hline$<40$ & $305763(10172)$ & $15.0 \%(14.9 \%$ to $15.1 \%)$ & Reference & \\
\hline $40-49$ & 274664 (8019) & $15.0 \%(14.9 \%$ to $15.1 \%)$ & 1.00 (0.99 to 1.02$)$ & 0.84 \\
\hline $50-59$ & $112623(3320)$ & $14.9 \%(14.7 \%$ to $15.1 \%)$ & 0.99 (0.97 to 1.02$)$ & 0.58 \\
\hline$\geq 60$ & $29949(1086)$ & $14.8 \%(14.4 \%$ to $15.3 \%)$ & 0.99 (0.95 to 1.03$)$ & 0.55 \\
\hline \multicolumn{5}{|l|}{ Total part B costs } \\
\hline \multicolumn{5}{|l|}{ Continuous ${ }^{\ddagger}$ : } \\
\hline For every 10 years & $780197(18956)$ & - & $2.4 \%(2.0 \%$ to $2.8 \%)$ & $<0.001$ \\
\hline \multicolumn{5}{|l|}{ Categorical: } \\
\hline$<40$ & $327707(10211)$ & $\$ 1008$ (\$1004 to $\$ 1012)$ & Reference & \\
\hline $40-49$ & 296680 (8071) & $\$ 1027(\$ 1022$ to $\$ 1032)$ & $1.8 \%$ (1.3\% to $2.4 \%)$ & $<0.001$ \\
\hline $50-59$ & $122758(3364)$ & $\$ 1056(\$ 1048$ to $\$ 1064)$ & $4.7 \%(3.8 \%$ to $5.6 \%)$ & $<0.001$ \\
\hline$\geq 60$ & 33052 (1111) & $\$ 1071$ (\$1055 to \$1088) & $6.3 \%(4.5 \%$ to $8.0 \%)$ & $<0.001$ \\
\hline
\end{tabular}


30 day mortality than those cared for by younger physicians, despite similar patient characteristics. These associations were found among physicians with low and medium volumes of patients but not among those with high volumes. Readmission rates and costs of care did not meaningfully vary with physician age. Taken together, our findings suggest that differences in practice patterns or process measures of quality between physicians with varying years of experience reported in previous studies ${ }^{1-437}$ might have a meaningful impact on patient outcomes.

Mechanisms that could explain our current findings can be broadly categorized into effects of age ("age effects") versus effects arising from differences in how younger and older physicians trained ("cohort effects"). It is possible that physicians further from training are less likely to adhere to evidence based guidelines, might use newly proved treatments less often, and might more often rely on clinical evidence that is not up to date. ${ }^{38}$ Moreover, while intense exposure to a large number of patients during residency training might enable physicians shortly out of such training to provide high quality care, the benefits of this training experience could wane if physicians care for fewer inpatients after residency. The lack of association between physician age and patient mortality among physicians with higher volume of patients supports this age related hypothesis.

Our findings might just as likely reflect cohort effects rather than declining clinical performance associated with greater age, which has important implications for interpretation of our findings. Hospital medicine is among the most rapidly evolving specialties within medicine, with dramatic changes in the training of recent cohorts of physicians who now work as hospitalists, including greater emphasis on multi-professional team based practice, adherence to clinical guidelines, training on patient handoffs, familiarity with shift work during residency training, and an improved hospital safety culture. Because the specialty of hospital medicine was first recognized in the 1990s, our study might have compared younger physicians who began their careers as hospitalists with older physicians who began their careers as primary care physicians and later became hospitalists. Thus, cohort differences in physician training, as well as declines in skill with aging, could explain our findings. Under this hypothesis, the cohort of physicians entering hospital medicine today might experience no reduction in patient outcomes with aging or possibly improved outcomes. Nonetheless, from the perspective of policymakers and administrators, current outcomes of older versus younger hospitalists might still be important to know irrespective of the path by which younger versus older physicians entered the specialty.

Our findings suggest that within the same hospital, patients treated by physicians aged $<40$ have 0.85 times the odds of dying (1.00/1.17) or an $11 \%$ lower probability of dying (10.8/12.1), compared with patients cared for by physicians aged $\geq 60$ (table 2 ). This difference in mortality is comparable with the impact of statins for the primary prevention of cardiovascular mortality on all cause mortality (odds ratio of 0.86 ) $^{39}$ or the impact of $\beta$ blockers on mortality among patients with myocardial infarction (incidence rate ratio of 0.86), ${ }^{40}$ indicating that our observed difference in mortality is not only statistically significant but arguably clinically significant. In addition, if our results are causal, an adjusted risk difference of 1.3 percentage points suggests that for every 77 patients treated by doctors aged $\geq 60$, one fewer patient would die within 30 days of admission if those patients were cared for by physicians aged $<40$.

\section{Policy implications}

Our findings should be regarded as exploratory. Nonetheless, they highlight the importance of patient outcomes as one component of an assessment of how physician practices change over a career. The purpose of continuing medical education is to ensure that physicians provide high quality care over the course of their careers. Although continuing medical education can take multiple forms that vary across specialties and across countries, the issue of ensuring that physicians keep up with current standards of care is applicable across all specialties and countries. In the US, for example, there are ongoing debates about the requirements for maintenance of certification, with many physicians arguing that current requirements could be burdensome and unneeded. Although our study did not analyze the effects of current such policies in the US, it suggests that continuing medical education of physicians could be important and that continual assessment of outcomes might be useful. In addition, although quality of care initiatives have largely focused on system level measures (such as hospital 30 day mortality and readmissions), there is increasing policy emphasis on the role of individual physicians in influencing costs and quality of care. ${ }^{41-43}$ For example, in the US, the Centers for Medicare and Medicaid Services has just promulgated draft final regulations for a new approach to pay individual clinicians for the value and quality of care they provide under the Medicare Access and CHIP Reauthorization Act (MACRA). ${ }^{44}$

\section{Strengths and limitations of study}

Our study has several limitations. First, our findings would be confounded if older physicians, on average, treat patients at higher risk of 30 day mortality because of factors unmeasured by our analysis. We specifically chose our within hospital study design to deal with this concern, hypothesizing that patients are essentially randomized to hospitalist physicians of various ages within the same hospital, an assumption supported by the largely similar demographic and clinical characteristics across patients that older and younger physicians treat. Second, we found that the positive association between physician age and patient mortality was driven primarily by physicians treating a low to medium volumes of patients, suggesting that high volumes could be "protective" of clinical skills. The association between practice volume and skills, however, could be bidirectional-physicians whose skills are 
declining might either self select, or be encouraged by others to leave, positions in which they are responsible for clinical management of large numbers of patients and could, therefore, treat fewer patients over time. Nonetheless, it is still important to know that older physicians with low and medium volumes of patients have worse patient outcomes because this information could suggest that specific interventions could be targeted towards these physicians. Third, the cross sectional nature of our study did not allow us to distinguish the degree to which our findings were attributable to declines in clinical performance with physician age versus cohort effects associated with secular changes in training. In the latter case, although older physicians could now be associated with higher patient mortality, as the current cohort of younger hospitalists age they might retain their superior patient outcomes even without individual maintenance of certification-type interventions. Fourth, physician age is only one of several factors associated with physician performance; physicians of varying skill level can be found within every age category. Finally, our findings might not generalize to the non-Medicare population, to patients cared for by surgeons or other specialists, or to physicians practicing in other countries (particularly as rates of hospitalist use might differ across countries). Further studies are warranted to understand whether similar patterns are observed in these other settings.

\section{Conclusions}

Patients in hospital treated by older hospitalists have higher mortality than patients cared for by younger hospitalists, except for hospitalist physicians with high volumes of patients. We found similar associations among patients treated by general internists. Readmission rates and costs of care did not meaningfully vary with physician age.

Contributors: All authors contributed to the design and conduct of the study, data collection and management, analysis interpretation of the data; and preparation, review, or approval of the manuscript. ABJ supervised the study and is guarantor.

Funding:YT is supported in part by the Abe Fellowship (Social Science Research Council and the Japan Foundation Center for Global Partnership). ABJ is supported by the Office of the Director, National Institutes of Health (NIH Early Independence Award, grant 1DP50D017897-01). The research conducted was independent of any involvement from the sponsors of the study. Study sponsors were not involved in study design, data interpretation, writing, or the decision to submit the article for publication.

Competing interests: All authors have completed the ICMJE uniform disclosure form at (available on request from the corresponding author) and declare: $A B$ J has received consulting fees unrelated to this work from Pfizer, Hill Rom Services, Bristol Myers Squibb, Novartis Pharmaceuticals, Vertex Pharmaceuticals, and Precision Health Economics, a company providing consulting services to the life sciences industry. JPN is a director of Aetna and has received consulting fees from EMD Serono; no other relationships or activities that could appear to have influenced the submitted work.

Ethical approval: The study was approved by the institutional review board at Harvard Medical School.

Data sharing: No additional data available.

Transparency statement: The corresponding author affirms that the manuscript is an honest, accurate, and transparent account of the study being reported; that no important aspects of the study have been omitted; and that any discrepancies are disclosed.
This is an Open Access article distributed in accordance with the Creative Commons Attribution Non Commercial (CC BY-NC 4.0) license, which permits others to distribute, remix, adapt, build upon this work non-commercially, and license their derivative works on different terms, provided the original work is properly cited and the use is non-commercial. See: http://creativecommons.org/licenses/ by-nc/4.0/.

1 Schwartz JS, Lewis CE, Clancy C, Kinosian MS, Radany MH, Koplan JP. Internists' practices in health promotion and disease prevention. A survey. Ann Intern Med 1991;114:46-53. doi:10.7326/0003-4819-114-1-46.

2 Czaja R, McFall SL, Warnecke RB, Ford L, Kaluzny AD. Preferences of community physicians for cancer screening guidelines. Ann Intern Med 1994;120:602-8.

doi:10.7326/0003-4819-120-7-199404010-00012

3 Fehrenbach SN, Budnitz DS, Gazmararian JA, Krumholz HM. Physician characteristics and the initiation of beta-adrenergic blocking agent therapy after acute myocardial infarction in a managed care population. Am J Manag Care 2001;7:717-23.

4 Choudhry NK, Fletcher RH, Soumerai SB. Systematic review: the relationship between clinical experience and quality of health care. Ann Intern Med 2005;142:260-73. doi:10.7326/0003-4819-142-4-200502150-00008.

5 O'Neill L, Lanska DJ, Hartz A. Surgeon characteristics associated with mortality and morbidity following carotid endarterectomy. Neurology 2000;55:773-81. doi:10.1212/WNL.55.6.773.

6 Southern WN, Bellin EY, Arnsten JH. Longer lengths of stay and higher risk of mortality among inpatients of physicians with more years in practice. Am J Med 2011;124:868-74. doi:10.1016/j. amimed 2011.04.011.

7 Epstein AJ, Srinivas SK, Nicholson S, Herrin J, Asch DA. Association between physicians' experience after training and maternal obstetrical outcomes: cohort study. BMJ 2013;346:f1596. doi:10.1136/bmj.f1596

8 Jena AB, Khullar D, Ho O, Olenski AR, Blumenthal DM. Sex Differences in Academic Rank in US Medical Schools in 2014. JAMA 2015;314:1149-58. doi:10.1001/jama.2015.10680.

9 Jena AB, Olenski AR, Blumenthal DM. Sex Differences in Physician Salary in US Public Medical Schools. JAMA Intern Med 2016;176:1294304. doi:10.1001/jamainternmed.2016.3284.

10 Tsugawa Y, Jena AB, Figueroa JF, Orav EJ, Blumenthal DM, Jha AK. Comparison of Hospital Mortality and Readmission Rates for Medicare Patients Treated by Male vs Female Physicians. JAMA Intern Med 2017;177:206-13. doi:10.1001/jamainternmed. 2016.7875 .

11 Tsugawa Y, Jena AB, Orav EJ, Jha AK. Quality of care delivered by general internists in US hospitals who graduated from foreign versus US medical schools: observational study. BMJ 2017;356:j273. doi:10.1136/bmj.j273.

12 Tsugawa Y, Jha AK, Newhouse JP, Zaslavsky AM, Jena AB. Variation in physician spending and association with patient outcomes. JAMA Intern Med 2017. doi:10.1001/jamainternmed.2017.0059.

13 McWilliams IM, Landon BE, Chernew ME, Zaslavsky AM. Changes in patients' experiences in Medicare Accountable Care Organizations. N Engl J Med 2014;371:1715-24. doi:10.1056/NEJMsa1406552.

14 Ross JS, Normand SL, Wang Y, et al. Hospital volume and 30-day mortality for three common medical conditions. N Engl J Med 2010;362:1110-8. doi:10.1056/NEJMsa0907130.

15 Drye EE, Normand SL, Wang Y, et al. Comparison of hospital risk-standardized mortality rates calculated by using in-hospital and 30-day models: an observational study with implications for hospital profiling. Ann Intern Med 2012;156:19-26. doi:10.7326/0003-4819-156-1-201201030-00004.

16 Temple RM, Kirthi V, Patterson LJ. Is it time for a new kind of hospital physician?BMJ 2012;344:e2240. doi:10.1136/bmj.e2240.

17 Wachter RM, Bell D. Renaissance of hospital generalists. BM 2012;344:e652. doi:10.1136/bmj.e652

18 Wachter RM, Goldman L. Zero to 50,000 - The 20th Anniversary of the Hospitalist. N Engl J Med 2016;375:1009-11. doi:10.1056/ NEJMp1607958.

19 Hinami K, Whelan CT, Miller JA, Wolosin RJ, Wetterneck TB. Society of Hospital Medicine Career Satisfaction Task Force. Job characteristics, satisfaction, and burnout across hospitalist practice models. J Hosp Med 2012;7:402-10. doi:10.1002/jhm.1907.

20 Kuo YF, Sharma G, Freeman JL, Goodwin JS. Growth in the care of older patients by hospitalists in the United States. N Engl I Med 2009;360:1102-12. doi:10.1056/NEJMsa0802381.

21 Research Data Assistance Center (ResDAC). Death Information in the Research Identifiable Medicare Data, 2016.

22 Elixhauser A, Steiner C, Harris DR, Coffey RM. Comorbidity measures for use with administrative data. Med Care 1998;36:8-27. doi:10.1097/00005650-199801000-00004.

23 Fitzmaurice GM, Laird NM, Ware JH. Applied longitudinal analysis.John Wiley \& Sons, 2012. 
24 Gardiner JC, Luo Z, Roman LA. Fixed effects, random effects and GEE: what are the differences?Stat Med 2009;28:221-39. doi:10.1002/ sim.3478.

25 Gunasekara FI, Richardson K, Carter K, Blakely T. Fixed effects analysis of repeated measures data. Int J Epidemiol 2014;43:264-9. doi:10.1093/ije/dyt221.

26 Arellano M. PRACTITIONERS'CORNER: Computing Robust Standard Errors for Within-groups Estimators. Oxf Bull Econ Stat 1987;49:431-4. doi:10.1111/j.1468-0084.1987.mp49004006.x.

27 Heinze G, Schemper M. A solution to the problem of separation in logistic regression. Stat Med 2002;21:2409-19. doi:10.1002/sim.1047.

28 Williams R. Using the margins command to estimate and interpret adjusted predictions and marginal effects. Stata / 2012;12:308-31. http://www.stata-journal.com/article.html?article=st0260

29 Halm EA, Lee C, Chassin MR. Is volume related to outcome in health care? A systematic review and methodologic critique of the literature. Ann Intern Med 2002;137:511-20. doi:10.7326/0003-4819-137-6200209170-00012.

30 Moscucci M, Share D, Smith D, et al. Relationship between operator volume and adverse outcome in contemporary percutaneous coronary intervention practice: an analysis of a quality-controlled multicenter percutaneous coronary intervention clinical database. I Am Coll Cardiol 2005;46:625-32. doi:10.1016/j.jacc.2005.05.048.

31 Nallamothu BK, Gurm HS, Ting HH, et al. Operator experience and carotid stenting outcomes in Medicare beneficiaries. JAMA 2011;306:1338-43. doi:10.1001/jama.2011.1357.

32 Joynt KE, Orav EJ, Jha AK. Physician volume, specialty, and outcomes of care for patients with heart failure. Circ Heart Fail 2013;6:890-7. doi:10.1161/CIRCHEARTFAILURE.112.000064.

33 Buntin MB, Zaslavsky AM. Too much ado about two-part models and transformation? Comparing methods of modeling Medicare expenditures. J Health Econ 2004;23:525-42. doi:10.1016/j. jhealeco.2003.10.005

34 Hanley JA, Negassa A, Edwardes MD, Forrester JE. Statistical analysis of correlated data using generalized estimating equations: an orientation. Am J Epidemiol 2003;157:364-75. doi:10.1093/aje/kwf215.
35 Lin DY, Psaty BM, Kronmal RA. Assessing the sensitivity of regression results to unmeasured confounders in observational studies. Biometrics 1998:54:948-63. doi:10.2307/2533848.

36 van Walraven C, Austin PC, Jennings A, Quan H, Forster AJ. A modification of the Elixhauser comorbidity measures into a point system for hospital death using administrative data. Med Care 2009:47:626-33. doi:10.1097/MLR.0b013e31819432e5.

37 Streja DA, Rabkin SW. Factors associated with implementation of preventive care measures in patients with diabetes mellitus. Arch Intern Med 1999:159:294-302. doi:10.1001/archinte.159.3.294.

38 Hlatky MA, Cotugno H, O'Connor C, Mark DB, Pryor DB, Califf RM. Adoption of thrombolytic therapy in the management of acute myocardial infarction. Am J Cardiol 1988;61:510-4 doi:10.1016/0002-9149(88)90755-2.

39 Taylor F, Huffman MD, Macedo AF, et al. Statins for the primary prevention of cardiovascular disease. Cochrane Database Syst Rev 2013; (1):CD004816

40 D’Ascenzo F, Taha S, Moretti C, et al. Meta-analysis of randomized controlled trials and adjusted observational results of use of clopidogrel, aspirin, and oral anticoagulants in patients undergoing percutaneous coronary intervention. Am J Cardiol 2015;115:1185-93. doi:10.1016/j.amjcard.2015.02.003.

41 Institute of Medicine. Variation in Health Care Spending: Target Decision Making, Not Geography. The National Academies Press, 2013.

42 Newhouse JP, Garber AM. Geographic variation in health care spending in the United States: insights from an Institute of Medicine report. JAMA 2013;310:1227-8. doi:10.1001/jama.2013.278139.

43 Newhouse JP, Garber AM. Geographic variation in Medicare services. N Engl/ Med 2013:368:1465-8. doi:10.1056/NEJMp1302981.

44 The Centers for Medicare \& Medicaid Services. Notice of proposed rule making: Quality Payment Program.The Center for Medicare \& Medicaid Services, 2016

Appendix: Supplementary tables 